\title{
Research Article \\ EPIDEMIOLOGY OF ACINETOBACTER SPECIES IN A TERTIARY CARE HOSPITAL WITH SPECIAL REFERENCE TO SEASONAL VARIATION
}

\section{GUPTA NEETU, GANDHAM NAGESWARI ${ }^{*}$, JADHAV SAVITA, MISRA RABINDRA NATH, ANGADI KALPANA AND VYAWAHARE CHANDA}

Department of Microbiology, Dr D.Y. Patil Medical College, Hospital and Research Centre, Dr D.Y. Patil Vidyapeeth, Pimpri, Pune, 411018, India

*Corresponding Author: Email-nageswari.rg@gmail.com

Received: June 18, 2018; Revised: June 26, 2018; Accepted: June 27, 2018; Published: June 30, 2018

\begin{abstract}
Background: The members of the genus Acinetobacter are persistent nosocomial pathogens. Strains with increased resistance to most $\beta$-lactam antibiotics, fluoroquinolones, and aminoglycosides continue to be detected worldwide. They are responsible for various nosocomial infections. This study aimed to find the prevalence, epidemiology, and changing trend of this organism in a tertiary care hospital over a period of three years. Materials and methods: Acinetobacter species isolated from various clinical samples of a hospital were studied. Susceptibility test was performed according to Clinical and Laboratory Standards Institute (CLSI) guidelines. Multidrug-resistant (MDR) strains and extended-spectrum beta-lactamases (ESBLs) were detected. Results: Out of 8,905 isolates, 490 (5.5\%) were Acinetobacter species. Maximum isolates $47.1 \%$ were obtained from intensive care units (ICUs). Off the MDR isolates, $87 \%$ were from the ICUs. All the ceftazidimeresistant isolates obtained from the outpatient department (OPD) were ESBL producers (100\%). Amongst the indoor wards paediatric ward showed the highest percentage of ESBL producers (71.4\%). The isolation rates throughout the three years were high in the late summer and monsoon, that is, may to oct. Conclusions: Isolation of MDR Acinetobacter species continues to be significant among ICUs. It is a major challenge to control infections caused by MDR Acinetobacter spp. Therefore, the continuous surveillance of the organism is needed to understand the changing trends in epidemiology and antibiotic resistance pattern.
\end{abstract}

Keywords- Acinetobacter spp, ICUs, Epidemiology, Multidrug resistance

Citation: Gupta Neetu, et al., (2018) Epidemiology of Acinetobacter Species in a Tertiary Care Hospital with Special Reference to Seasonal Variation. International Journal of Microbiology Research, ISSN: 0975-5276 \& E-ISSN: 0975-9174, Volume 10, Issue 6, pp.-1283-1286.

Copyright: Copyright@2018 Gupta Neetu, et al., This is an open-access article distributed under the terms of the Creative Commons Attribution License, which permits unrestricted use, distribution and reproduction in any medium, provided the original author and source are credited.

\section{Introduction}

The increasing role of Acinetobacter spp. in the establishment of nosocomial infections is a worldwide concern. The Progressive development of resistance to several antimicrobial agents used in the treatment poses a major challenge in health care [1]. In a study by the Global Burden of Diseases, mortality due to infections, in general, has decreased in the past few years but it still remains a major factor [2]. Acinetobacter spp. are primarily opportunistic pathogens that affect critical care units; however, it is gradually becoming a persistent nosocomial agent in other wards. Once established, remains endemic in the institutions and causes sequential outbreaks [3]. The ubiquitous presence of Acinetobacter poses a challenge in eradicating it from the hospital environment. According to recent studies, weather plays an additional epidemiological determinant in the incidence of these hospital associated infections [4]. However, in developing countries there is a lack in the published data on the seasonal trends of these pathogens. Therefore, this study aimed to find the prevalence of Acinetobacter in a tertiary care hospital over the past three years, to understand its distribution in the different areas of the hospital and different samples; to understand the changing trends in the epidemiology and antibiotic resistance patterns of antibiotics in various wards.

\section{Material and Methods}

The study period extended from January 2014 to December 2016 and involved the collection of continuous data of Acinetobacter spp. isolated from various samples received in the Department of Microbiology. The samples obtained from the patients admitted and treated in the various wards and ICUs of Dr D Y Patil Medical College, Hospital, and Research Centre, Dr D Y Patil Vidyapeeth, Pune,
India, were included in the study. Various samples such as blood, urine, sputum, pus, and other body fluids received in the laboratory were processed. The growth of genus Acinetobacter on agar plates was identified by colony morphology, Gram stain, positive catalase test, negative oxidase test, the absence of motility, and standard biochemical reactions $[1,5]$. Antimicrobial susceptibility testing of the isolates was performed by Kirby-Bauer disc diffusion method for ciprofloxacin $(5$ $\mu \mathrm{g})$, ceftazidime $(30 \mu \mathrm{g})$, cefotaxime $(30 \mu \mathrm{g})$, imipenem $(10 \mu \mathrm{g})$, amikacin (30 $\mu \mathrm{g})$, gentamicin $(10 \mu \mathrm{g})$, ampicillin $(10 \mu \mathrm{g})$, and cotrimoxazole $(25 \mu \mathrm{g})$ discs (HiMedia, Mumbai, India). Zone size was interpreted according to CLSI guidelines $[6,7]$. Multidrug-resistant (MDR) strains (the isolates resistant to at least one agent in three or more categories of drugs) were detected using antibiotics from different categories $[8,9]$. Extended-spectrum beta-lactamase (ESBL) production in Acinetobacter spp. was detected in the isolates showing reduced susceptibility to ceftazidime by double disc approximation test performed on Mueller-Hinton agar plates using the discs of ceftazidime alone, ceftazidime-clavulanic, and ceftazidime-tazobactam [10-12]. To determine the seasonal trends in the isolation rate of Acinetobacter sps., we analyzed the monthly isolation rate from different samples and wards.

\section{Results}

During the study period, a total of 34,910 samples were processed and 8,905 isolates were obtained. Out of these isolates, 490 (5.5\%) were Acinetobacter spp. In ward-wise distribution, the maximum number of isolates were obtained from ICUs 231 (47.1\%), followed by surgery 94 (19.1\%), medicine 89 (18.1\%), pediatric $29(5.91 \%)$ and gynecology wards $15(3.06 \%)$,,[Table-1]. 


\begin{tabular}{|c|c|c|c|c|c|c|c|}
\hline \multirow{2}{*}{$\begin{array}{l}\text { Place of } \\
\text { isolation }\end{array}$} & \multicolumn{7}{|c|}{ Three years (2014 to 2016) } \\
\hline & Blood & Pus & Urine & Respiratory & C.S.F & Body Fluid & Total \\
\hline ICUs & 50 & 5 & 4 & 134 & 2 & 36 & 231 \\
\hline Surgery & 3 & 76 & 1 & 0 & 0 & 14 & 94 \\
\hline Medicine & 29 & 6 & 1 & 41 & 1 & 11 & 89 \\
\hline Pediatric & 26 & 1 & 1 & 1 & 0 & 0 & 29 \\
\hline Gynecology & 3 & 11 & 0 & 0 & 0 & 1 & 15 \\
\hline Others & 1 & 14 & 0 & 0 & 0 & 13 & 28 \\
\hline OPD & 1 & 3 & 0 & 0 & 0 & 0 & 4 \\
\hline Total & 113 & 116 & 7 & 176 & 3 & 75 & 490 \\
\hline
\end{tabular}

Table-2 Antibiotic resistance patterns in Acinetobacter isolates

\begin{tabular}{|c|c|c|c|c|c|c|c|c|}
\hline Antibiotics & ICUs & Surgery & Med. & Gynec. & Pediatric & OPD & $\begin{array}{l}\text { Other } \\
\text { wards }\end{array}$ & Total \\
\hline Ampicillin $(10 \mu \mathrm{g})$ & $98.2 \%$ & $93.5 \%$ & $92 \%$ & $100 \%$ & $89.2 \%$ & $100 \%$ & $92.8 \%$ & $95.4 \%$ \\
\hline Ceftazidime $(30 \mu \mathrm{g})$ & $95.2 \%$ & $88.2 \%$ & $91 \%$ & $100 \%$ & $72.4 \%$ & $75 \%$ & $75 \%$ & $90.6 \%$ \\
\hline Cefotaxime $(30 \mu \mathrm{g})$ & $93 \%$ & $82.9 \%$ & $67.4 \%$ & $86.6 \%$ & $65.5 \%$ & $75 \%$ & $78.5 \%$ & $83.6 \%$ \\
\hline Imipenem $(10 \mu \mathrm{g})$ & $17.7 \%$ & $50 \%$ & $16.8 \%$ & $20 \%$ & $3.4 \%$ & $0 \%$ & $17.8 \%$ & $22.8 \%$ \\
\hline Amikacin $(30 \mu \mathrm{g})$ & $75.7 \%$ & $70.21 \%$ & $32.5 \%$ & $66.6 \%$ & $17.8 \%$ & $0 \%$ & $67.8 \%$ & $62 \%$ \\
\hline Gentamicin $(10 \mu \mathrm{g})$ & $77 \%$ & $63.8 \%$ & $42.6 \%$ & $73.3 \%$ & $17.2 \%$ & $0 \%$ & $60.7 \%$ & $63 \%$ \\
\hline Ciprofloxacin $(5 \mu \mathrm{g})$ & $84.4 \%$ & $79.7 \%$ & $47.1 \%$ & $73.3 \%$ & $24.1 \%$ & $0 \%$ & $67.8 \%$ & $71.2 \%$ \\
\hline $\begin{array}{l}\text { Trimethoprim sulfamethoxazole } \\
(1.25 / 23.75 \mu \mathrm{g})\end{array}$ & $81.6 \%$ & $79.7 \%$ & $59.5 \%$ & $80 \%$ & $37.9 \%$ & $25 \%$ & $57.1 \%$ & $72.7 \%$ \\
\hline
\end{tabular}

Table-3 Percentage of MDR ( $n=386 / 490)$ and ESBL (189/444) Acinetobacter spp. from different wards

\begin{tabular}{|l|c|c|}
\hline \multicolumn{1}{|c|}{ Place of isolation } & MDR isolates & ESBL isolates \\
\hline ICUs & $87 \%$ & $43.6 \%$ \\
\hline Surgery & $85.1 \%$ & $20.4 \%$ \\
\hline Medicine & $66.2 \%$ & $50 \%$ \\
\hline Pediatric & $41.3 \%$ & $71.4 \%$ \\
\hline Gynecology & $80 \%$ & $53.3 \%$ \\
\hline Others & $75 \%$ & $45.4 \%$ \\
\hline OPD & $25 \%$ & $100 \%$ \\
\hline Total & $78.7 \%$ & $42.5 \%$ \\
\hline
\end{tabular}

Table-4 Frequency of isolates in descending order in three years

\begin{tabular}{|c|c|c|}
\hline 2014 n=3242 (Percentage) & $2015 n=2844$ (Percentage) & $2016 n=2819$ (Percentage) \\
\hline S.aureus $998(30.7 \%)$ & S. aureus $888(31.2 \%)$ & S. aureus $687(24.3 \%)$ \\
\hline Klebsiella spp.564 (17.3\%) & E. coli $571(20 \%)$ & Klebsiella spp. $528(18.7 \%)$ \\
\hline E. coli $484(14.9 \%)$ & Klebsiella spp.444 (15.6\%) & E. coli $499(17.7 \%)$ \\
\hline P.aeruginosa $272(8.3 \%)$ & P.aeruginosa $275(9.6 \%)$ & P. aeruginosa $266(9.4 \%)$ \\
\hline Citrobacter spp.230 (7.09\%) & Citrobacter spp. 195(6.8\%) & Acinetobacter spp.181(6.4\%) \\
\hline Enterococcus spp.197 (6.07\%) & Acinetobacter spp.169 (5.9\%) & Enterococcus spp. 169 (5.9\%) \\
\hline Acinetobacter spp.140 (4.3\%) & Enterococcus spp.120 (4.2\%) & Citrobacter spp.132 (4.6\%) \\
\hline Remaining 357 (11\%) & Remaining $182(6.3 \%)$ & Remaining 357 (12.6\%) \\
\hline
\end{tabular}

Table-5 Seasonal variation in isolation of Acinetobacter species from clinical samples

\begin{tabular}{|l|c|c|c|c|c|c|c|c|c|c|c|c|c|c|c|}
\hline Clinical Samples & Jan & Feb & Mar & Apr & \multicolumn{1}{|c|}{ May } & \multicolumn{1}{|l|}{ Jun } & Jul & Aug & Sep & Oct & Nov & Dec & Total \\
\hline Blood & 7 & 10 & 6 & 0 & 2 & 4 & 29 & 16 & 20 & 6 & 7 & 6 & 113 \\
\hline Urine & 1 & 1 & 2 & 1 & 0 & 0 & 1 & 0 & 0 & 0 & 1 & 0 & 7 \\
\hline Respiratory sample & 5 & 20 & 10 & 16 & 19 & 8 & 19 & 17 & 19 & 21 & 8 & 14 & 176 \\
\hline Pus & 5 & 3 & 3 & 12 & 13 & 16 & 16 & 12 & 10 & 9 & 12 & 5 & 116 \\
\hline Body fluids & 2 & 5 & 3 & 7 & 4 & 11 & 8 & 9 & 8 & 14 & 2 & 5 & 78 \\
\hline Total & 20 & 39 & 24 & 36 & 38 & 39 & 73 & 54 & 57 & 50 & 30 & 30 & 490 \\
\hline
\end{tabular}

Table-6 Seasonal variation in isolation rates of Acinetobacter species in various wards and ICUS

\begin{tabular}{|c|c|c|c|c|c|c|c|c|c|c|c|c|c|}
\hline Wards and ICUs & Jan & Feb & Mar & Apr & May & Jun & Jul & Aug & Sep & Oct & Nov & Dec & Total \\
\hline ICUs & 10 & 23 & 13 & 16 & 13 & 10 & 30 & 28 & 26 & 30 & 12 & 20 & 231 \\
\hline Medicine wards & 5 & 13 & 7 & 4 & 14 & 6 & 22 & 15 & 19 & 6 & 6 & 5 & 122 \\
\hline Surgery wards & 4 & 3 & 4 & 16 & 11 & 23 & 20 & 11 & 11 & 14 & 11 & 5 & 133 \\
\hline OPD & 1 & & & & & & 1 & & 1 & & 1 & & 4 \\
\hline Total & 20 & 39 & 24 & 36 & 38 & 39 & 73 & 54 & 57 & 50 & 30 & 30 & 490 \\
\hline
\end{tabular}

The sample-wise distribution of the isolates revealed that the maximum number of isolates were obtained from the respiratory samples $176(35.9 \%)$ followed by pus $116(23.6 \%)$, blood $113(23 \%)$, body fluids including C.S.F 78(15.9\%) and urine 7 (1.4\%), [Table-1]. The maximum isolates from respiratory samples were obtained from ICUs (134/176), 76.1\% followed by medicine ward (41/176), 23.2\%. In addition, maximum isolates from blood samples were obtained from ICUs (50/113), 44.2\%, followed by medicine (29/113), 25.6\% and pediatric ward
(26/113), 23\%. Whereas, maximum pus isolates were obtained from surgery ward (76/116), 65.5\%, followed by gynecology ward (11/116), 9.4\%, [Table-1]. A gender-wise distribution of Acinetobacter isolates revealed male predominance in all wards except pediatric ward where the number of both sex was almost same (14-male,15-female). Overall distribution showed male: female ratio of 1.9:1. Isolates from the samples of gynecology ward showed $100 \%$ resistance to ampicillin and ceftazidime. 
Strains isolated from ICUs samples showed high resistance to ampicillin, ceftazidime, cefotaxime, ciprofloxacin and cotrimoxazole (above 80\%). Similarly, in other wards also maximum resistance to these antibiotics observed. In ICUs and wards imipenem is the most sensitive drug followed by amikacin and gentamicin [Table-2]. Out of the 490 Acinetobacter isolates, 386 (78.7\%) were MDR organisms. Maximum MDR organisms were isolated from ICUs (201/231), $87 \%$, followed by surgery (80/94), 85.1\%, gynecology (12/15), 80\%, medicine (59/89), $66.2 \%$, pediatric (12/29), $41.3 \%$, and OPD (1/4), $25 \%$. The isolation rate from the remaining wards was (21/28) $75 \%$, [Table-3]. Out of 490 Acinetobacter isolates, $444(90.6 \%)$ showed reduced susceptibility to ceftazidime, which was further confirmed for ESBL production using the double disc approximation test. Out of 444 ceftazidime-resistant isolates, 189 (42.5\%) were confirmed as ESBL producers. Ceftazidime-resistant Acinetobacter isolates from OPD were all ESBL producers (3/3), 100\%. In the hospitalized patients, the maximum Acinetobacter isolates were obtained from pediatric ward (15/21), 71.4\%, followed by gynecology (8/15), 53.3\%, medicine (40/80), 50\%, and surgery wards (17/23), $20.4 \%$. Maximum ESBL producers were detected using $\beta$-lactamase inhibitor tazobactam (181/189), 95.7\% compared with clavulanic acid (59/189), 31.2\%, [Table-3]. Yearwise distribution revealed that maximum isolates were obtained from ICUs in the year 2015. However, in the surgery and medicine wards, the isolation was higher in 2014. The isolation rate of Acinetobacter spp. was the highest in 2016. Table-4 shows the occurrence of Acinetobacter spp. in the order of frequency during the three years. The isolation of Acinetobacter spp. was higher in the summer and rainy seasons (May to Oct) in these three years [Fig-1]. Sample-wise distribution revealed seasonal peak in two major type of infections blood stream infections (July-Sep) months and pyogenic infection (Apr-Nov). Three cases of meningitis were there in these three years which were in the months of June-July-august [Table-5]. While in the ward-wise isolation, seasonal trend was seen in ICUs with highest isolation rate during the months of July-oct, Medicine wards in July-Sep and in surgery ward extended from the month of Apr-Nov [Table 6]. Seasonal trend was not observed in the isolation of MDR and non-MDR isolates.

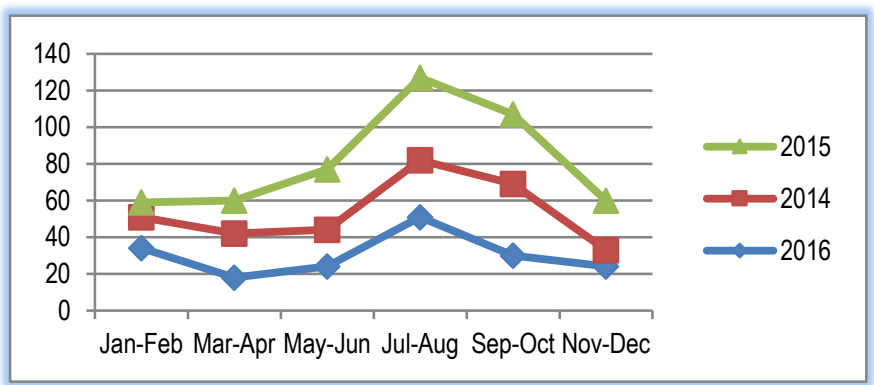

Fig-1 Trend of monthly and yearly isolation of Acinetobacter

\section{Discussion}

In this study, out of total 8,905 isolated organisms, 490 (5.5\%) were Acinetobacter. Kaur, et al. found $10.2 \%$ prevalence of Acinetobacter in Bathinda, India, Dash, et al. found $3 \%$ prevalence in Odisha, India, and Uwingabiye, et al. found $6.94 \%$ prevalence of all bacterial isolates in Morocco $[9,13,14]$. The maximum isolates of Acinetobacter were obtained from ICUs (47.1\%), followed by surgery (19.1\%) and medicine wards (18.1\%). In the study of Jaggi, et al., out of the total Acinetobacter isolates, $76.7 \%$ were obtained from ICUs [15]. The isolation rate is highest in ICUs due to the existing environment and risk factors for the persistence of this nosocomial pathogen. These consist of frequently admitted immunocompromised patients with multiple comorbidities, colonization followed by infection, prolonged stay of patients which leads to cross-infection among the patients, prolonged use of broad-spectrum antibiotics and indwelling devices. In this study, the maximum isolation of Acinetobacter spp. was from respiratory samples (35.9\%), followed by pus $(23.6 \%)$, blood $(23 \%)$, body fluids $(15.3 \%)$, and urine $(1.4 \%)$. Similarly, in the study of Jaggi, et al., maximum isolates of Acinetobacter were obtained from respiratory samples (57.4\%), followed by blood (23.8\%) and pus (13.5\%) [15]. In addition, Uwingabiye, et al. obtained maximum isolates of Acinetobacter spp. from bronchopulmonary samples (44.67\%), followed by blood (14.51\%) and deep pus $(12.47 \%)$ [14]. Hospital-associated infections are most likely to involve the respiratory tract followed by urinary tract and may progress to septicemia $[16,17]$. In this study, urine isolates were less frequent compared with others. Among the isolates in respiratory samples received, maximum isolates were from ICUs (76.1\%), followed by medicine (23.2\%). Further among isolates in blood samples received, maximum was from ICUs $(44.2 \%)$, followed by medicine $(25.6 \%)$ and pediatric wards $(23 \%)$. While among isolates from pus samples received, maximum isolates were from surgery ward $(65.5 \%)$, followed by gynecology ward $(9.4 \%)$. The factors responsible for a higher isolation rate in the respiratory and blood samples received from ICUs and pus samples received from surgery and gynecology wards are mechanical ventilation, nasogastric tubing, the disruption of anatomical barriers because of the use of invasive devices, surgeries, impaired immunological response, and ability of this organism to survive in high fluid content. Acinetobacter spp. were isolated more frequently from samples of male $(59.1 \%)$ patients than that of female patients $(40.8 \%)$, which is concords other studies [18]. The susceptibility testing results revealed that Acinetobacter spp. were resistant to most frequently used antibiotics. The isolates from ICUs and other wards showed the highest resistance to ampicillin (95.4\%), followed by ceftazidime $(90.6 \%$ ) and cefotaxime (83.6\%), similar to the results of other studies and lowest resistance to imipenem (22.8\%), followed by amikacin $(62 \%)$, and gentamicin $(63 \%)[12,19]$. Overall, a high resistance pattern was observed in ICUs, followed by surgery and gynecology wards. This study found a prevalence of $78.7 \%$ MDR strains; however, Begum Shahzeera, et al. in Islamabad reported $100 \%$ prevalence of MDR A. baumannii strains [20]. A meta-analysis conducted by Bialvaei, et al. revealed that the prevalence of MDR A. baumannii is $72 \%$ in Iranian population [21]. Maximum MDR strains were found in samples received from ICUs (87\%), followed by surgery (85.1\%) and gynecology wards (80\%) and least in the samples received from pediatric ward (41.3\%). Acinetobacter and MDR Acinetobacter spp. are persistent pathogens in ICUs. In the gynecology ward, although the number of isolated strains of Acinetobacter spp. was low, maximum strains obtained were MDR. The sample-wise distribution, revealed that maximum MDR Acinetobacter strains were obtained from respiratory samples $(88.6 \%)$, followed by pus samples $(85.3 \%)$ and minimum from blood samples (55.7\%). In addition, Haung, et al. found maximum MDR strains from respiratory samples [22].

In this study, $42.5 \%$ of ceftazidime-resistant isolates were ESBL producers. In a study conducted by Banerjee, et al. in $2013,50.7 \%$ of the Acinetobacter isolates were ESBL-producing, and Sinha, et al., in 2007 , detected $28 \%$ isolates to be ESBL-producing [18,23]. Maximum ESBL producers were detected by screening positive isolates obtained from OPD (100\%). During ward-wise and sample-wise distribution, maximum ESBL producers were detected from the pediatric ward and blood samples, respectively. This finding is not consistent with other studies that reported a trend of occurrence of ESBL producers similar to that of the detection of the MDR isolates $[18,24]$. In this study, the maximum MDR strains were detected in the respiratory sample received from ICUs, whereas maximum ESBL producers were detected in the blood samples, followed by respiratory samples, received from pediatric ward. The reason for this trend is not clear. It was observed that the isolation frequency of Acinetobacter spp. is increasing over the years. From being the seventh commonest isolate in 2014, it became the fifth commonest isolate in 2016. In addition, this upward trend of isolation has also been reported in other studies [3]. The reasons for this increasing trend are multifactorial such as the endemic presence of the organism in the environment, development of multidrug-resistant strains, and longer hospital stay leading to colonization $[3,25,26]$. Coming to the discussion of seasonal trends, there are two main studies on the isolation of Acinetobacter based on seasonality performed by CDC. These studies observed higher rate of infection during the months of JulyOctober than November to June. In this study, we found seasonal variation in case of Blood stream infection, pyogenic infection, meningitis cases and not in other infections. This could be due to increased moisture and warmth in the environment, leading to an increase in their growth. Furthermore, skin colonization is higher in temperate climates than cold climates [27]. Therefore, measures to prevent skin colonization need to be more stringently followed during these months. While observing the seasonal trend in different hospital areas, trend seen in all ICUs, Medicine wards and surgery wards. 
It is not surprising as the organism survive both in dry and moist conditions and hospital environment serve as reservoir where critical patients are lying but many studies including molecular need to be performed to understand the mechanism of seasonal variation.

\section{Conclusion}

In conclusion, Acinetobacter spp. can spread easily and remain endemic in hospitals. Therefore, the epidemiological profile of this organism should be monitored and any change in the trend must be highlighted. The infection control measures could include environmental surveillance and detection of colonization. The isolation of a patient (colonized or infected) with MDR is necessary.

Application of research: Continuous training of staff regarding the correct aseptic care of indwelling devices mainly vascular catheters and endotracheal tubes and extensive surveillance during summer and rainy seasons are the optimal approaches to eliminate Acinetobacter spp. from the hospital environment. As the isolation of MDR and ESBL Acinetobacter spp. is very high, the detection of $M D R$ and ESBL producers is necessary to prevent therapeutic failures.

\section{Research category: Epidemiology of Acinetobacter}

Acknowledgement / Funding: Authors are thankful to Department of Microbiology Dr D Y Patil Medical College, Hospital and Research Centre, Dr D Y Patil Vidyapeeth, Pimpri, Pune, 411018

\section{*Principal Investigator: Dr Neetu Gupta}

University: Dr D Y Patil Vidyapeeth, Pimpri, Pune, 411018

\section{Author Contributions: All authors contributed equally.}

Author Statement: All authors read, reviewed, agreed and approved the final manuscript

\section{Conflict of interest: None declared}

Ethical approval: This article does not contain any studies with human participants or animals performed by any of the authors

\section{References}

[1] Anitha M., Monisha D.M., Mohamed AS., Pandurangan S. (2016) Sch. Acad. J. Biosci, 4(4A), 335-41.

[2] Ghanshani R., Gupta R., Gupta B.S., Kalra S, Khedar R.S., Sood S. (2015) Lung India, 32(5), 441-8.

[3] Morfín-Otero R., Alcántar-Curiel M.D., Rocha M.J., C.M. AlpucheAranda C.M., Santos-Preciado J.I., Gayosso-Vázquez C et al., (2013) Chemotherapy,59, 57-65.

[4] Caldeiral S.M., Cunha A.R da., Akazawa R.T., Moreira R.G., Rosário de Souza $L$ do R de., Fortaleza Carlos Magno Castelo C.B. (2015) Rev. Saúde Pública ,49, 19.

[5] Lone R., Shah A., Kadri S.M., Lone S., Shah F. (2009) Bangladesh J Med Microbiol, 03, 34-8.

[6] Clinical and Laboratory Standards Institute. M100-S23. Wayne, PA, USA: CLSI; 2013

[7] Clinical and Laboratory Standards Institute. M100-S25. Wayne, PA, USA: CLSI; 2015.

[8] Magiorakos A.P., Srinivasan A., Carey R.B., Carmeli Y, Falagas M.E., Giske C.G., et al., (2012) Clin Microbiol Infect, 18, 268-81.

[9] Kaur A., Gill A.k., Singh S., Kaur N., Mahajan A., Mittal V. (2016) Int J Health Sci Res, 6(6), 83-9.

[10] Singla P., Sikka R., Deeep Antariksh., Gagneja D., Chaudhary U. (2014) J Clin Diag Res, 8(4) ,16-9.

[11] Pandey A. (2009) Indian J Pathol Microbiol, 52(3), 456-7.

[12] Dhara M., Disha P., Sachin P., Manisha J., Seema B., Vega M.M.
(2012) National J Med Re, 2(3), 348-53.

[13] Dash M., Padhi S., Patnaik S., Mohantyl, Misra P. (2013) Avicenna J Med, 3(4), 97-102.

[14] Uwingabiye J., Frikh M., Lemnouer A., Bssaibis F., Belefquih B., Maleb A., et al., (2016) Pan Afr Med J, 23, 191.

[15] Jaggi N., Sissodia P., Sharma L. (2012) J Microbiol Infect Dis, 2(2), 57-63.

[16] Bergogne-Berezin E. and Towner K.J. (1996) Clin. Microbiol. Rev, 9 , $148-65$.

[17] Cisneros J.M., Reyes M.J., Pachon J., Becerril B., Caballero F.J., Gracia-Garmendia., et al., (1996) Clin Infect Dis, 22,1026-1032.

[18] Banerjee M., Chaudhary B.L., Shukla S. (2015) IJSR.NET. 2015, 4(6), 1183-6.

[19] Gupta N., Gandham N., Jadhav S., Misra R.N. (2015) J Nat Sc Biol Med, 6(1), 159-62.

[20] Begum S., Hasan F., Hussain S., Shah A.A. (2013) Pak J Med Sci, 29(5), 1253-8.

[21] Bialvaei A.Z., Kouhsari E., Abargouei AS., Amirmozafari N., Ramazanzadeh R., Ghadimi-Dareajini A., et al., (2017) J Chemother, 1-9

[22] Gandham P. (2014) Int. J. Microbiol. App. Sci, 3(2), 9-13.

[23] Sinha M., Srinivase H., Macaden R. (2007) Indian J Med Re, 126, 637.

[24] Nath H., Barkataki D. (2016) Int. J. Curr. Microbiol. App. Sci, 5(11), 515-22.

[25] Gandham N.R., Gupta N., Jadhav S.V., Misra R.N. (2012) Med. J DY Patil Univ, 5(2), 151-3.

[26] Baang J.H., Axelrod P., Decker B.K., Hujer A.M., Dash G., Truant A.R., et a., (2012) Am J Infect Control, 40(2), 134-7.

[27] Alan M., Klompas M. (2010) J Clin Outcomes Manag, 17(8), 363-9. 